\title{
Correction to: All-cause mortality and disease progression in SARS-CoV-2-infected patients with or without antibiotic therapy: an analysis of the LEOSS cohort
}

\author{
Maximilian J. Schons ${ }^{1} \cdot$ Amke Caliebe $^{2,3} \cdot$ Christoph D. Spinner $^{4} \cdot$ Annika Y. Classen $^{1,5} \cdot$ Lisa Pilgram $^{6}$. \\ Maria M. Ruethrich ${ }^{7}$. Jan Rupp ${ }^{8}$. Susana M. Nunes de Miranda ${ }^{1}$. Christoph Römmele ${ }^{9} \cdot$ Janne Vehreschild $^{1,5,6}$. \\ Bjoern-Erik Jensen ${ }^{10}$. Maria Vehreschild ${ }^{6}$. Christian Degenhardt ${ }^{11}$. Stefan Borgmann ${ }^{12} \cdot$ Martin Hower $^{13}$. \\ Frank Hanses ${ }^{14}$. Martina Haselberger ${ }^{15}$. Anette K. Friedrichs ${ }^{16}$ on behalf of the LEOSS-study group
}

Published online: 15 December 2021

(c) The Author(s) 2021

\section{Correction to: Infection https://doi.org/10.1007/s15010-021-01699-2}

The original version of this article unfortunately contained a mistake.

In this article the author name Susana M. Nunes de Miranda was incorrectly written as Susana Nunes de Miranda.

The original article has been corrected.

The original article can be found online at https://doi.org/10.1007/ s15010-021-01699-2.

\section{Anette K. Friedrichs}

Anette.Friedrichs@uksh.de

Maximilian J. Schons

Maximilian.schons@uk-koeln.de

Amke Caliebe

caliebe@medinfo.uni-kiel.de

Christoph D. Spinner

christoph.spinner@mri.tum.de

Annika Y. Classen

annika.classen@uk-koeln.de

\section{Lisa Pilgram}

lisa.pilgram@kgu.de

Maria M. Ruethrich

Maria.Ruethrich@med.uni-jena.de

Jan Rupp

Jan.Rupp@uksh.de

Susana M. Nunes de Miranda

susana.nunes-de-miranda@uk-koeln.de

Christoph Römmele

christoph.roemmele@uk-augsburg.de
Open Access This article is licensed under a Creative Commons Attribution 4.0 International License, which permits use, sharing, adaptation, distribution and reproduction in any medium or format, as long as you give appropriate credit to the original author(s) and the source, provide a link to the Creative Commons licence, and indicate if changes were made. The images or other third party material in this article are included in the article's Creative Commons licence, unless indicated otherwise in a credit line to the material. If material is not included in the article's Creative Commons licence and your intended use is not permitted by statutory regulation or exceeds the permitted use, you will need to obtain permission directly from the copyright holder. To view a copy of this licence, visit http://creativecommons.org/licenses/by/4.0/.

Janne Vehreschild

joerg.vehreschild@uk-koeln.de

Bjoern-Erik Jensen

Bjoern-Erikole.Jensen@med.uni-duesseldorf.de

Maria Vehreschild

Maria.Vehreschild@kgu.de

Christian Degenhardt

christian.degenhardt@klinikum-karlsruhe.de

Stefan Borgmann

stefan.borgmann@klinikum-ingolstadt.de

Martin Hower

Martin.Hower@klinikumdo.de

Frank Hanses

Frank.Hanses@klinik.uni-regensburg.de

Martina Haselberger

martina.haselberger@klinikum-passau.de

1 Department I of Internal Medicine, University Hospital of Cologne, Cologne, Germany

2 Institute for Medical Informatics and Statistics, University Hospital Schleswig-Holstein, Campus Kiel, Kiel, Germany 
3 Kiel University, Kiel, Germany

4 School of Medicine, Department of Internal Medicine II, Technical University of Munich, University Hospital Rechts Der Isar, Munich, Germany

5 German Centre for Infection Research (DZIF), Partner Site Bonn-Cologne, Cologne, Germany

6 Department II of Internal Medicine, Hematology/Oncology, Goethe University, Frankfurt, Frankfurt am Main, Germany

7 Institute for Infection Medicine and Hospital Hygiene, University Hospital Jena, Jena, Germany

8 University Hospital Schleswig-Holstein, Lübeck, Germany

$9 \quad$ Internal Medicine III - Gastroenterology and Infectious Diseases, University Hospital of Augsburg, Augsburg, Germany

10 Clinic for Gastroenterology, Hepatology and Infectiology, University Hospital Düsseldorf, Heinrich-Heine-University Düsseldorf, Düsseldorf, Germany
11 Municipal Hospital Karlsruhe, Karlsruhe, Germany

12 Department of Infectious Diseases and Infection Control, Ingolstadt Hospital, Ingolstadt, Germany

13 Department of Pneumology, Infectious Diseases and Intensive Care, Klinikum Dortmund gGmbH, Dortmund, Germany

14 Interdisciplinary Emergency Department, University Hospital Regensburg, Regensburg, Germany

15 Department of Internal Medicine I, Passau Hospital, Passau, Germany

16 Clinic for Internal Medicine I, University Hospital Schleswig-Holstein, Campus Kiel, Kiel, Germany 